\title{
Improved Modelling of Tool Tracking Errors by Modelling Dependent Marker Errors
}

\author{
Stephen Thompson, Graeme Penney, Prokar Dasgupta, and David Hawkes
}

\begin{abstract}
Accurate understanding of equipment tracking error is essential for decision making in image guided surgery. For tools tracked using markers attached to a rigid body, existing error estimation methods use the assumption that the individual marker errors are independent random variables. This assumption is not valid for all tracking systems. This paper presents a method to estimate a more accurate tracking error function, consisting of a systematic and random component. The proposed method does not require detailed knowledge of the tracking system physics. Results from a pointer calibration are used to demonstrate that the proposed method provides a better match to observed results than the existing state of the art. A simulation of the pointer calibration process is then used to show that existing methods can underestimate the pointer calibration error by a factor of two. A further simulation of laparoscopic camera tracking is used to show that existing methods can not model important variations in system performance due to the angular arrangement of the tracking markers. Arranging the markers such that the systematic errors are nearly identical for all markers, the rotational component of the tracking error can be reduced, resulting in a significant reduction in target tracking errors.
\end{abstract}

Index Terms-Point Based Tracking, FLE, TRE.

\section{INTRODUCTION}

$\mathrm{T}$ HE accurate tracking of localiser probes, surgical tools, and cameras is essential for most image guided surgery. Currently available image guided surgery systems, for example the StealthStation Surgical Navigation System (Medtronic, Inc., Minneapolis, $\mathrm{MN}$ ) and the VectorVision System (BrainLAB AG, Feldkirchen, Germany) rely on optical tracking cameras to track tools using an array of tracked markers. These have the advantage that the markers themselves can be very accurately tracked (typically to within $0.2 \mathrm{~mm}$ for active markers). Optical tracking systems have the disadvantage that the tracking markers cannot usually be placed at the tip of the instrument. The position of the tip of the instrument is determined by a rigid body registration using prior knowledge (determined using a calibration process) of the tracking geometry. As long as the distance from the tracking markers to the instrument

- Copyright (c) 2010 IEEE. Personal use of this material is permitted. However, permission to use this material for any other purposes must be obtained from the IEEE by sending a request to pubs-permissions@ieee.org.

- S. Thompson and D. Hawkes are with the Centre for Medical Image Computing,UCL.E-mail: s.thompson@ucl.ac.uk

- G. Penney is with Imaging Sciences, KCL.

- P. Dasgupta is with the Department of Urology Guys and St Thomas Hospital.

- This work was funded by EU FP7 PASSPORT Project, EPSRC DTA Funding, Prostate Action, and the Guys and St Thomas Charity. This work was also partly supported by the UCLH/UCL Comprehensive Biomedical Research Centre (CBRC) (Grant Ref. 96). This work was partly undertaken at UCLH/UCL who received a proportion of funding from the Department of Healths NIHR Biomedical Research Centres funding scheme.

Manuscript received June 27, 2012; revised August 29, 2012. tip remains relatively short, for example in neurosurgery or open surgery, optical tracking works well, usually with errors at the tool tip under a millimetre.

During abdominal laparoscopic surgery the surgeon has less tactile feedback and a limited field of view. Therefore image guidance has the potential to bring great benefits to laparoscopic surgery, possibly opening up surgical routes not hitherto available to the surgeon. However, if as is the case for laparoscopic surgery, the tools or indeed the laparoscope are long, the errors at the tool or laparoscope tip can be vary large.

The inability to track either the tools or the laparoscope with sufficient accuracy is one of the problems that has to date prevented the development of image guidance systems for laparoscopic surgery. Historically three approaches have been used for tracking laparoscopic tools. Kinematic tracking of the laparoscopic tool chain, for example in the daVinci robot [1], has so far proven insufficiently accurate due to the build up of errors along the kinematic chain. Electromagnetic tracking systems should in theory allow the tip of the laparoscope to be tracked directly, avoiding the problems associated with the long laparoscopic tool. To date however they have not been shown to work in a laparoscopic application, probably due to the practicalities of getting the base unit near enough to the laparoscopic tip and free from interference. If infra red emitting diodes (IRED)s can be localised to within $0.2 \mathrm{~mm}$ and placed in a well spread pattern on the exposed end of the laparoscope then it should be possible, in theory and assuming that the individual errors are independent, [2], to achieve sub millimetre accuracy at the tool tip for some practical applications. Such accuracy however has not to date been achieved in any practical laparoscopic application. This paper aims to show that the reason for this is not 
that the reported IRED localisation error of $0.2 \mathrm{~mm}$ is overly optimistic, but rather that in certain cases the individual IRED errors are not independent.

This paper puts forward a simple empirical method to determine the marker localisation error function for a widely used optical tracking system (Optotrak Certus, Northern Digital, Ontario Canada). The method avoids making assumptions about the marker localisation error nor is any knowledge of the physics of the camera system required. Therefore the method should be directly applicable to other similar tracking systems. With the IRED localisation error functions known it is possible to model the tracking of a laparoscope to enable development of systems with improved accuracy. Using the empirically derived error function this paper shows that when designing a tracking collar for a laparoscope, the angular orientation of the IREDs is of critical importance. Specifically, and somewhat counter intuitively, a collar where all the IREDs share the same orientation will be significantly more accurate than a collar with IREDs arranged at varying angles.

The remainder of this paper is structured as follows. Section 2 further explains the motivation of the paper and Section 3 introduces and reviews the existing state of the art for estimating the tracking error. In Section 4 the data used in the paper are described. Section 5 defines the terms used in the paper and gives an overview of the point based registration methods used for rigid body tracking.

The data and methods used in this paper are based on the problem of pointer calibration. This application was chosen for two reasons. Firstly the calibration and use of pointers is common for most image guided interventions. Secondly, it is likely that marker localisation errors have a dominant impact on the observable calibration errors. In contrast validation using laparoscopic camera tracking data is more difficult due to potential errors in the camera calibration and video processing masking errors due to IRED localisation. However the methods and conclusions of this paper are applicable to the tracking of any tool, laparoscope, or ultrasound probe, using similar tracking systems.

The methods used for estimating the IRED localisation error functions are then introduced together with methods for validation. A results section follows in which the estimated IRED localisation error functions are presented and validated. Section 8 uses the derived error functions to model both the pointer calibration and laparoscope tracking processes. In both cases the results are of great practical importance for image guided laparoscopic surgery. The paper is concluded with a discussion of the various results.

\section{Background AND Motivation}

This paper is motivated by the desire to bring image guidance to laparoscopic surgery. Image guidance should help mitigate the effects of the loss of tactile feedback and the limited field of view during laparoscopic

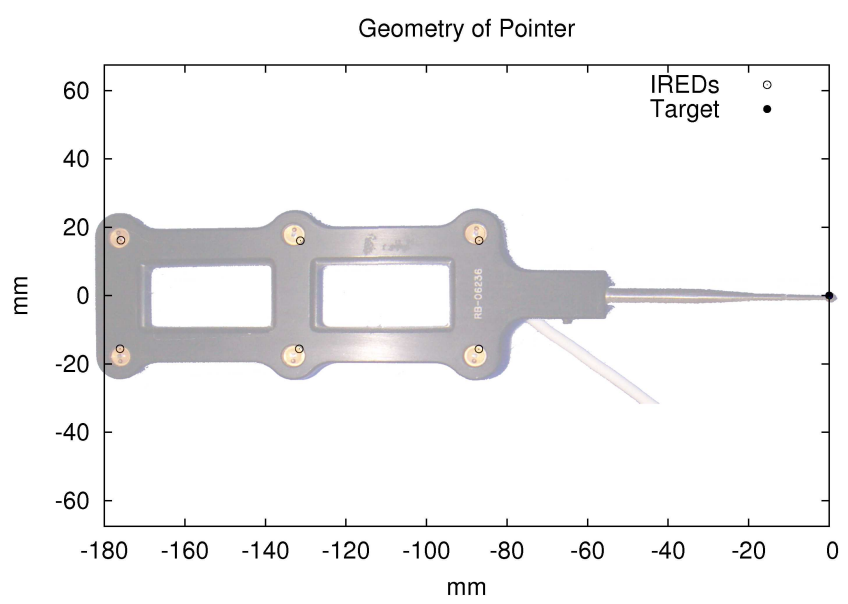

Fig. 1. A pointer (Northern Digital, Ontario Canada) used for point localisation during image guided surgery. The position of the pointer tip is estimated from the measured positions of the 6 markers (IREDs), under the assumption that the pointer forms a rigid body.

surgery. Furthermore as the surgical scene is viewed through a video screen, laparoscopic surgery lends its self to image guidance and augmented reality as images can be directly overlaid on to the existing video display. In spite of this, image guidance for laparoscopic surgery is not yet a reality.

One barrier to the implementation of image guided laparoscopic surgery is the requirement to track the laparoscopic camera and tools accurately. During open surgery or neurosurgery where the tools are relatively short this has been successfully achieved using optical tracking systems.

Figure 1 shows a typical pointer tool for image guided surgery. The tip of the pointer can be used to localise a set of fiducial markers or to localise a point of surgical interest.

The error at the tool tip will be determined by the accuracy with which each of the 6 markers are tracked (the marker localisation error) and the geometry of the markers relative to the pointer tip. Under the assumption that the marker localisation error is independent, homogeneous, and isotropic, the error at the tool tip can be estimated using the formula put forward by Fitzpatrick et al. [2].

$$
\frac{\left\langle T R E^{2}\right\rangle}{\left\langle F L E^{2}\right\rangle} \approx \frac{1}{N}\left(1+\frac{1}{3} \sum_{k=1}^{3} \frac{d_{k}^{2}}{f_{k}^{2}}\right)
$$

The terms in equation 1 are defined as follows.

- TRE is the Target Registration Error. In the case of a tracked pointer it refers to the error in locating the pointer tip using the measured positions of the 6 IREDs.

- FLE is the Fiducial Localisation Error. In the case of the tracked pointer it can refer to the error in measuring the position of each IRED. It is important 
to point out that the error models developed in this paper are specific to the application of IRED localisation, and may not be applicable to the more general case of fiducial localisation. Nonetheless, to maintain continuity with the literature the term fiducial localisation error (FLE) will be used throughout this paper.

- $N$ refers to the number of fiducial markers, for example in the case of the tracked pointer shown in figure $1 N=6$

- $k$ is the direction of the measurement $(x, y, z)$.

- $d^{2}$ and $f^{2}$ are the second moments of inertia (about the centroid of the fiducial points) of the target point and the fiducial points respectively.

Based on equation 1, for an Optotrak Certus system with a marker localisation error of $0.20 \mathrm{~mm}$ RMS (based on [3], [4]) the error (TRE) at the tip of the standard pointer (Figure 1) will be $0.25 \mathrm{~mm}$ which is negligibly small for the application. Indeed, even if equation 1 was wrong by a factor of 2 (due to a violation of the assumed distribution of FLE) the additional target registration error (TRE) of $0.25 \mathrm{~mm}$ would be of little practical interest.

The tracking geometry for laparoscopic procedures is significantly more challenging. For the pointer shown in Figure 1 the distance from the centroid of the IREDs to the target (pointer tip) is approximately $130 \mathrm{~mm}$. For a laparoscopic camera the distance from the IRED centroid to the target can be as much as $600 \mathrm{~mm}$. This additional length amplifies the TRE along with any errors in the estimation of TRE. For laparoscopic applications accurate modelling of FLE, and hence the TRE, becomes of great practical importance.

There is a significant body of work, which is discussed in the next section, extending the work of [2] to non isotropic errors and more arbitrary error distributions. However none of the approaches address the issue that the IRED localisation errors are not independent. It is well known, see [3] and [5], that the localisation error for an individual IRED depends on the IRED's position and pose. In the case of an object tracked with a set of IREDs mounted to a single rigid body the individual IRED errors therefore cannot be independent. Later in this paper, see Figure 16, we present an example where using the erroneous assumption of independent FLE can overestimate the tracking error for a laparoscope by around $2 \mathrm{~mm}$, large enough to be clinically significant. In another application, pointer calibration, see Figure 13 , the use of an independent marker error model can underestimate the calibration error by a factor of 2 .

This paper presents a simple method for estimating the dependent, arbitrarily distributed IRED localisation errors for a given tracking system. Accurate modelling of the IRED tracking error enables accurate modelling of the target (pointer tip or laparoscope lens) tracking error. Furthermore it will enable improved tracker arrangements to be designed, the accurate testing of improved point registration algorithms [6], compensation for systematic tracking errors, and the use of probabilistic motion filters to improve the tracking accuracy.

\section{State of the Art}

Estimation of the target tracking accuracy of a system can be done in one of two complementary ways. The first is to measure the system accuracy directly. The second is to determine the component errors and then derive the system accuracy from the component errors.

\subsection{Direct Measurement}

The most straightforward way to asses the accuracy of a tracking system is to perform a direct experimental evaluation. A good example for the case of camera tracking is given by [7]. In this case a grid of known geometry is imaged with the complete system and the overlay error measured directly. A similar procedure could be applied to a tracked pointer if the position of a set of divots were measured independently with a sufficiently accurate measurement system.

Experimental measurement of the system errors has several drawbacks. Firstly there is no standard way of performing the measurement. Therefore it is extremely difficult to compare results across the literature. Secondly, the measured errors will have several sources, that cannot be easily separated. For example small errors and eccentricity in the manufacture of the divots would add to the measured errors. An experimentally measured error is therefore the sum of the pointer tip tracking error and any experimental errors. Separating these error sources is not usually possible. Lastly, it is difficult to make the experimental measurement representative of the conditions during surgery.

\subsection{System Error from Component Error}

An alternative approach is to derive the system error from the component errors. This has been done numerically by [8] and [9]. Numerical simulation is also used by [2] and [10] to validate their analytical derivations. Numerical modelling has the benefit that it is straightforward to model tracking systems of arbitrary geometry, arbitrary error sources, and arbitrary models of the error sources. This modelling enables potential tracking systems to be evaluated and compared easily, see [5].

Analytical models have the advantage that they allow rapid determination of the target tracking error, however they are at present limited to certain classes of component errors. Fitzpatrick et al. [2] provides the earliest and most widely used model of target localisation error, based on an assumption of independent, homogeneous and isotropic marker localisation noise. Wiles et al. [10] extended this work for independent, homogeneous, but anisotropic noise. Mohghari and Abolmaesumi [11] introduced analytical methods to account for inhomogeneous noise, and [12] give a general first order solution for TRE for anisotropic and inhomogeneous FLE. 
These methods all depend on an assumption of independent marker errors. For the Optotrak Certus, however, it is known that the FLE will depend on the position, pose, and velocity of the IRED, [3]. For instrument tracking where several IREDs are attached to a single rigid body these parameters will not be independent, hence the FLE will not be independent. Despite this, estimating the accuracy of surgical systems or tools based on these assumptions is commonplace in the literature and in many cases the results are satisfactory. See [13], [5], and [14] for examples.

\subsection{Determination of the IRED Localisation Error}

Wiles et al. [3] and Barnes et al. [15] both demonstrate methods to measure the IRED localisation error relying on a second measurement system (a coordinate measuring machine) that has a higher accuracy than the optical tracking system. This has two disadvantages. Firstly, such a secondary measurement system is not typically available to the user of the tracking system. Therefore the user is usually reliant on the results of a characterisation performed by the tracking system manufacturer. In the case of the Optotrak Certus the accuracy is reported as $0.2 \mathrm{~mm}$ root mean square (RMS) across the "Characterised Region". ${ }^{1}$ Secondly, it is difficult to perform such measurements over the full range of conditions that may occur in use. For example, the measurements performed by [15] are necessarily limited to a very small region of interest and do not take into account any changes in the orientation of the IRED. Nonetheless they do make the important point that the accuracy of the system is inversely related to the speed of the tracked object.

Bauer et al. [16] present an analytical method for estimating the tracking error for a marker being tracked by multiple cameras. Their approach, however, requires a level of knowledge of the camera system optics that would be beyond the level of a typical user of a commercial tracking system.

\subsubsection{Fiducial Localisation Error from Fiducial Registra- tion Error}

An alternative approach is to estimate the marker localisation error using the fiducial registration error (FRE). The FRE is defined in [17] as the difference between the position of a fiducial estimated by a rigid body registration and the measured position of the fiducial, denoted here as $\mathbf{x}_{\mathbf{r b}}$ and $\mathbf{x}_{\mathbf{m}}$ respectively. Equation 2 defines the FRE for a given fiducial.

$$
F R E=\mathbf{x}_{\mathbf{r b}}-\mathbf{x}_{\mathbf{m}}
$$

For the case of isotropic, independent, and homogeneous FLE, [2] derived equation 3, where $N$ refers to the number of fiducial markers.

$$
\left\langle F R E^{2}\right\rangle=\left(1-\frac{2}{N}\right)\left\langle F L E^{2}\right\rangle
$$

1. The tracking cameras are designed to operate to the specified accuracy with a certain volume. If the tracked objects leave this volume they may still be tracked, but with significantly higher errors.
Equation 3 can be rearranged to estimate FLE given the measurable FRE.

An extension to equation 3 to the case of independent, anisotropic and inhomogeneous FLE was derived by [17]. In this case the covariance matrix of the FLE at each fiducial marker is estimated using the covariance of the FRE at the marker and a geometry dependent matrix. The form of the matrix is quite complex so will not be repeated here, equation 4 gives a general representation of the equation, where $\Sigma_{F L E_{a}}$ and $\Sigma_{F R E_{a}}$ represent the covariances of the FLE and the FRE at marker $a$. The matrix $A$ is a function of the geometry of the rigid body to which the fiducial is attached. See [17] for full details.

$$
\Sigma_{F L E_{a}}=A^{-1} \Sigma_{F R E_{a}}
$$

Equation 4 provides a useful way to estimate the FLE based on the FRE, which can be measured easily for a given application. However, equation 4 depends on the assumption of independent FLE. There is a danger when tracking a rigid body on which the FLE is not independent, if all the markers are subject to the same, dependent, error then the FRE will not reflect the true errors. In this case equation 4 will significantly underestimate the true FLE.

\subsection{Summary of Current Methods}

Existing methods to estimate the FLE for a typical optical tracking system depend on either a high level of knowledge of the tracking system physics, specialist equipment not typically available to the users of these systems, or overly simplistic assumptions about the distribution of FLE. In this paper a simple approach to estimating the marker localisation error function is presented. The next section introduces the experimental data used to quantify the system accuracy in this paper.

\section{DATA}

Two sets of rigid body tracking data are used in this study. A standard pointer (Figure 1) consisting of 6 IREDs with a $3 \mathrm{~mm}$ diameter ball attached to the tip was tracked whilst the pointer was pivoted in a stationary divot. The rigid body was swept through an angle of approximately 120 degrees in two directions. An Optotrak Certus camera system, see Figure 2, was used to record the position of each of the 6 IREDs. Each frame of data consists of the measured position of each of the 6 IREDs in the Optotrak's coordinate system, defined in Figure 2. 5838 frames were captured. Table 1 lists the range of the relevant IRED parameters. The speed was measured in $\mathrm{mm}$ per frame and converted to $\mathrm{mm}$ per second using a measured average frame rate of 30 frames per second.

To enable the sampling of errors with a dependence on IRED orientation a second rigid body was rigidly attached to the pointer and the process repeated. Figure 4 shows the rigid body attached to the pointer from Figure 1 . The combined rigid body consisted of 22 IREDs orientated at various angles. 5530 frames were captured. 


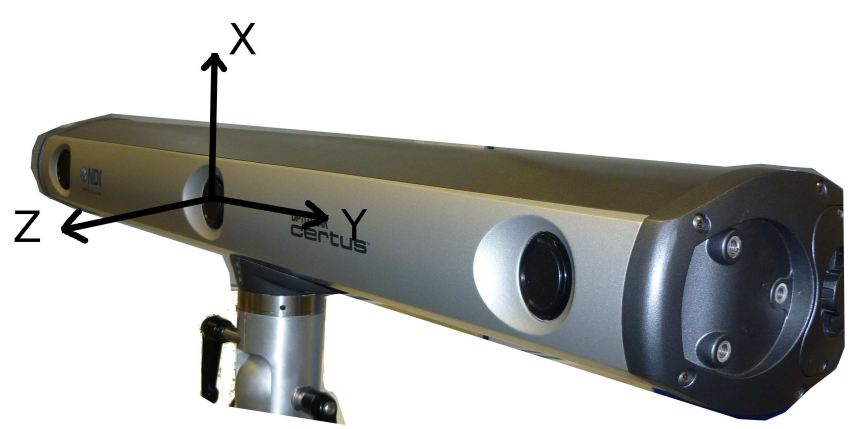

Fig. 2. The coordinate system of the Optotrak Certus. The $z$ axis is aligned with the surface normal of the central camera.
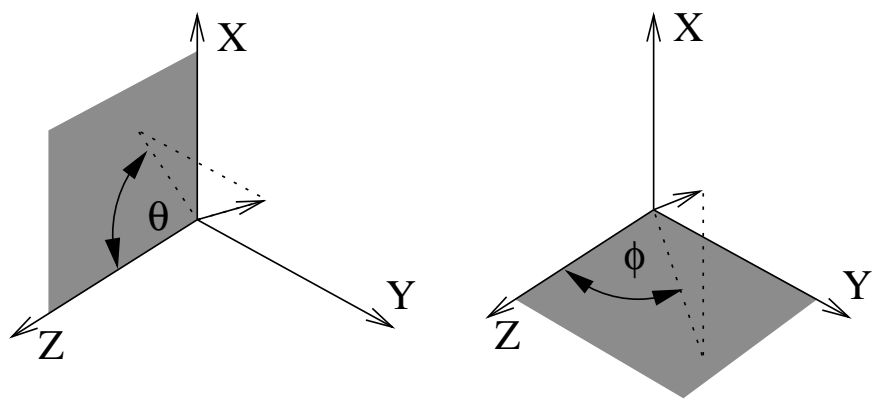

Fig. 3. The definition of the IRED surface normal angles in the coordinate system of the Optotrak Certus. $\theta$ is the angle projected onto the $X Z$ plane. $\phi$ is the angle projected onto the $\mathrm{YZ}$ plane.

\begin{tabular}{|l|l|l|l|l|l|l|}
\hline & $\begin{array}{l}\mathrm{x} \\
(\mathrm{mm})\end{array}$ & $\begin{array}{l}\mathrm{y} \\
(\mathrm{mm})\end{array}$ & $\begin{array}{l}\mathrm{z} \\
(\mathrm{mm})\end{array}$ & $\theta\left(^{0}\right)$ & $\phi\left(^{0}\right)$ & $\begin{array}{l}\mathrm{s} \\
(\mathrm{mm} / \mathrm{s})\end{array}$ \\
\hline Max & -166 & 114 & -2081 & 50 & 33 & 1995 \\
Min & -318 & -145 & -2336 & -68 & -66 & 0 \\
Mean & -235 & -12 & -2206 & -4 & -18 & 42 \\
$\sigma$ & 36 & 48 & 46 & 22 & 23 & 38 \\
\hline
\end{tabular}

TABLE 1

The range of parameters for the 5838 tracking experiments undertaken using the 6 IRED pointer shown in figure 1 with the camera system shown in figure 2 .

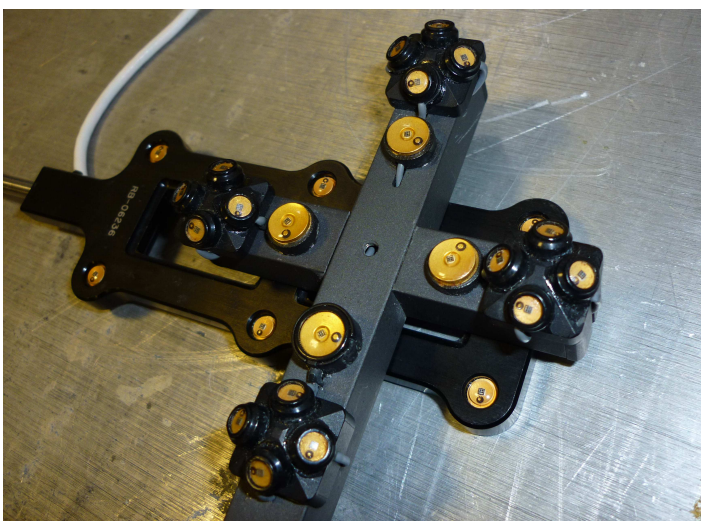

Fig. 4. The composite rigid body, consisting of the 6 IRED pointer with a 20 IRED cross rigidly fixed to it. Four of the IREDs on the cross were faulty, giving a composite rigid body with 22 effective IREDs.

\begin{tabular}{|l|l|l|l|l|l|l|}
\hline & $\begin{array}{l}\mathrm{x} \\
(\mathrm{mm})\end{array}$ & $\begin{array}{l}\mathrm{y} \\
(\mathrm{mm})\end{array}$ & $\begin{array}{l}\mathrm{Z} \\
(\mathrm{mm})\end{array}$ & $\theta\left(^{0}\right)$ & $\phi\left({ }^{0}\right)$ & $\begin{array}{l}\mathrm{S} \\
(\mathrm{mm} / \mathrm{s})\end{array}$ \\
\hline Max & -153 & 115 & -2078 & 65 & 59 & 341 \\
Min & -300 & -146 & -2325 & -72 & -74 & 0 \\
Mean & -216 & -21 & -2208 & -13 & -9 & 61 \\
$\sigma$ & 30 & 51 & 44 & 25 & 26 & 39 \\
\hline
\end{tabular}

TABLE 2

The range of parameters for the 5530 tracking experiments undertaken with the 22 IRED composite pointer shown in figure 4 .

Each frame consists of the measured position of the 22 IREDs. Table 2 lists the range of the relevant IRED parameters. The speed was measured in $\mathrm{mm}$ per frame and converted to $\mathrm{mm}$ per second using a measured average frame rate of 30 frames per second.

\section{Methods}

The common assumption in the literature is that the FLE can be modelled as an independent random variable in each principal axis of the tracking camera. Typically the random variable is normally distributed but this is not necessarily the case. In this paper the assumption is made that the FLE can be better modelled as a sum of a systematic error and a random variable, i.e. $F L E_{X, Y, Z}=f_{X, Y, Z}+\mathcal{N}\left(0, \sigma_{X, Y, Z}\right)$. For the Optotrak Certus it is known that the error is dependent on the position and angular orientation of a given IRED, [3], and the speed of motion of the IRED, [15]. The FLE function can therefore be written as in equation 5

$$
F L E_{X, Y, Z}=f_{X, Y, Z}(\theta, \phi, x, y, z, s)+\mathcal{N}\left(0, \sigma_{X, Y, Z}\right)
$$

where $x, y$, and $z$ define the position of the IRED in the coordinate space of the camera system. $\theta$ and $\phi$ define the pose of the IRED, based on the angle between the IRED's surface normal, see figure 5 , and the $Z Y$ and $Z X$ planes respectively, see Figure $3 . s$ is the speed of motion, defined as the magnitude of the distance between the current position of the IRED and its last measured position, divided by the elapsed time between the position measurements.

The random variable term $\mathcal{N}\left(0, \sigma_{X, Y, Z}\right)$ accounts for the "jitter", which is the noise in the measurement for a static IRED. It is likely that the value of the standard deviation is also dependent on the position and speed of the IRED, though, for simplicity, this has not been modelled.

In this paper the functions $f_{X, Y, Z}$ and standard deviations $\sigma_{X, Y, Z}$ are estimated using the measured position of the IREDs on the rigid bodies. This will be discussed in section 5.3. Prior to that it is helpful to define the point based registration method and pointer calibration method used.

\subsection{Point Based Registration}

Throughout this paper rigid body point based registrations are performed using the orthogonal Procrustes for- 


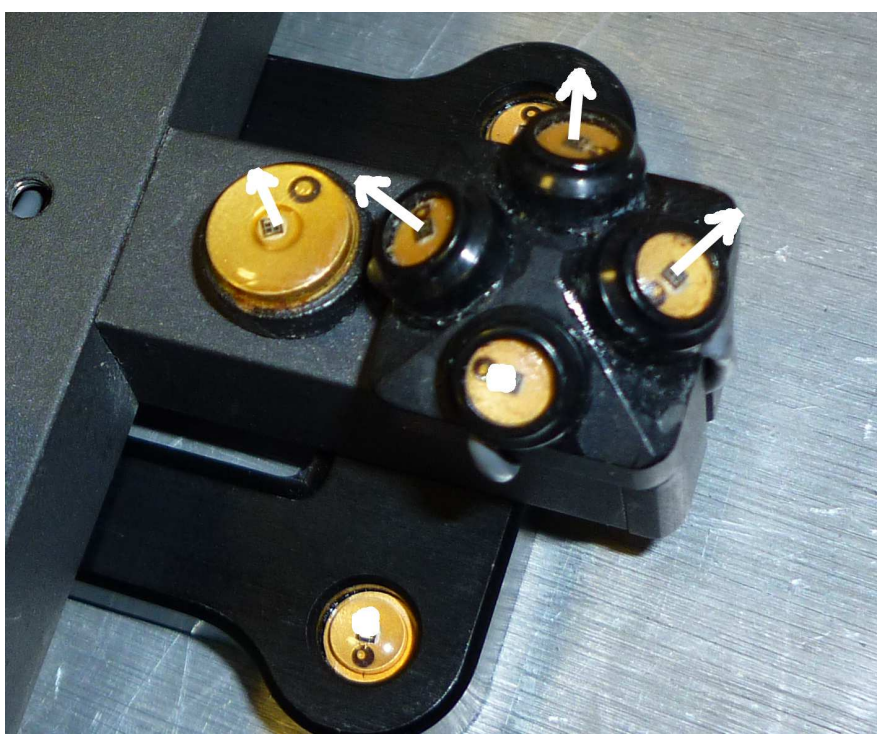

Fig. 5. An example of surface normal vectors on the cross rigid body

mulation introduced by [18]. The registration transform is then found using singular value decomposition, see [19]. Orthogonal Procrustes and singular value decomposition will give an optimal registration when the FLE is independent, homogeneous, isotropic, and normally distributed. As discussed this is known not to be the case for this application. However, the algorithm is widely used, robust, and fast. The implications of using it are discussed further at the end of this paper in Section 9.

\subsection{Pointer Calibration}

The pointer calibration process used in this paper is as follows. The pointer tip is placed in a shallow divot and held in the divot by downward pressure whilst the pointer is moved through a range of angles. The range of angles is limited by the ability of the tracking cameras to detect the IRED positions. In practice it was possible to achieve rotations about each axes of approximately 60 degrees before IRED visibility was compromised. The location of the $m$ visible IREDs is recorded for each of the $n$ frames of data, to give $m \times n$ 3D coordinates $\mathbf{x}_{\mathbf{i}, \mathbf{j}}$ for each IRED $i$ and each frame $j$.

\subsubsection{Defining the Reference Rigid Body}

If the assumption underlying this paper (that IRED errors are dependent on the position and attitude of the IRED) is correct then the apparent shape of the rigid body may change as it moves. Therefore when defining a reference body it is important to use data representative of the tracking task, in this case a pointer calibration. The following process was used.

For each rigid body a set of $m$ points $\left(X_{r e f, 0}\right)$ was defined based on a physical measurement of the rigid body. This measurement does not need to be particularly accurate, values within about $5 \mathrm{~mm}$ of the true position will suffice. The rigid body transform between each frame $\left(X_{j}\right)$ and the reference frame is found using the method described in Section 5.1. The IREDs in frame $j$ are then transformed into the coordinates of the reference frame using the transform thus found. For each IRED this gives $n$ position estimates in the coordinates of the reference rigid body. The average of these positions is used to define a new reference rigid body $\left(X_{r e f, 1}\right)$. The process is repeated until convergence of the average IRED positions was achieved, i.e. $\left(X_{r e f, i+1} \approx X_{r e f, i}\right)$. In the cases presented here convergence occurred within 3 to 4 iterations.

\subsubsection{Calibrating the Pointer}

For each frame of calibration data the transform to the reference rigid body was found as per Section 5.1. For the full data set this gives $n 3 \times 3$ rotation matrices $\left(R_{i}\right)$ and $n 3$ vectors $\mathbf{T}_{\mathbf{i}}$. During the calibration procedure the tip of the pointer (denoted $\mathbf{p}$ in the pointer's coordinate system) is constrained to pass through the divot centre (denoted $\mathbf{q}$ in the global coordinate system). This yields equation 6 for each frame of data.

$$
\mathbf{q} \approx\left[\mathbf{R}_{\mathbf{i}}\right] \mathbf{p}+\mathbf{T}_{\mathbf{i}}
$$

Equation 6 can be rearranged and concatenated for all $n$ frames of data. The least squares solution for $\mathbf{p}$ and $\mathbf{q}$ can then be found using equation 7 .

$$
\left\{\begin{array}{l}
\mathbf{q} \\
\mathbf{p}
\end{array}\right\}=\left[\begin{array}{cc}
n\left[I_{3}\right] & -\sum_{i=1}^{n}\left[R_{i}\right] \\
-\sum_{i=1}^{n}\left[R_{i}\right]^{T} & n\left[I_{3}\right]
\end{array}\right]^{-1}\left\{\begin{array}{c}
\sum_{i=1}^{n} \mathbf{T}_{\mathbf{i}} \\
-\sum_{i=1}^{n}\left[R_{i}\right]^{T} \mathbf{T}_{\mathbf{i}}
\end{array}\right\}
$$

\subsubsection{Assessing the Calibration Error}

If the position of the pointer tip is known (denoted $\mathbf{p}_{\mathrm{gs}}$, for gold standard), then the calibration error is defined by 8 .

$$
E_{C a l}=\{\mathbf{p}\}-\left\{\mathbf{p}_{\mathrm{gs}}\right\}
$$

In general however $\mathbf{p}_{\mathrm{gs}}$ is not known. In the case of the pointer calibration there is no practical method that could determine the pointer tip position more accurately than the presented calibration method. In lieu of knowing the actual pointer tip position the spread of the estimated pointer tip positions about an estimated divot location is often used as a proxy for the calibration error. Equation 9 defines the pointer tip spread for an individual frame of data.

$$
P T S=\left[R_{i}\right] \mathbf{p}+\mathbf{T}_{\mathbf{i}}-\mathbf{q}
$$

The RMS value of PTS is often measured, however it is well known that this value is not a predictor of the actual calibration error, as will be demonstrated in Section 8.1. 


\subsection{Estimating IRED Localisation Error Using Leave One Out Registration}

The FLE functions, equation 5, are estimated by fitting functions to estimated FLEs. The first stage in this process is estimating the individual FLEs. For a given IRED, if its actual position $\left(\mathrm{x}_{\mathrm{gs}}\right)$ were known, the FLE for a given measurement of its position $\left(\mathrm{x}_{\mathbf{m}}\right)$ could be found simply by subtracting the measured position from the actual position, as per equation 10 .

$$
\mathrm{FLE}=\mathrm{x}_{\mathrm{m}}-\mathrm{x}_{\mathrm{gs}}
$$

In some situations it may be possible to know $\mathbf{x}_{\mathbf{g s}}$ accurately enough for this method to be used, for example [3] and [15]. However characterising the tracking system over the full range of positions, angles, and speeds that may be used in practice would be extremely time consuming and impractical.

The approach used here is to replace the $\mathbf{x}_{\mathbf{g s}}$ with the position of the IRED estimated by performing a rigid body registration using the other IREDs on the rigid body. For a rigid body with $n$ IREDs the position of a given IRED $i$ can be estimated by finding the transform to the reference rigid body. The $i^{\prime} t h$ IRED on the reference is then transformed by the inverse of this transform to give an estimate of the actual position of

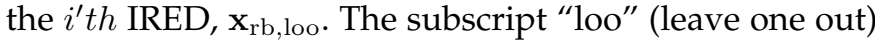
being used to differentiate the estimate from $\mathbf{x}_{\mathbf{r b}}$ used in equation 2, where the IREDs own measured position is used in the rigid body registration. The FLE can then be expressed as in 11.

$$
\mathbf{F L E}=\mathbf{x}_{\mathbf{m}}-\mathrm{x}_{\mathrm{rb}, \text { loo }}+\mathrm{TRE}
$$

Two approaches to estimating this error are used in this paper. Equation 11 is split into its systematic and random components. To yield equation

$$
\mathrm{FLE}_{\text {sys }}+\mathrm{FLE}_{\mathrm{ran}}=\mathrm{x}_{\mathrm{m}}-\mathrm{x}_{\mathrm{rb}, \mathrm{loo}}+\mathrm{TRE}_{\mathrm{sys}}+\mathrm{TRE}_{\mathrm{ran}}
$$

\subsection{Estimation of Systematic Errors}

Estimation of the systematic errors $\left(\mathbf{F L E}_{\mathrm{sys}}\right)$ relies on the assumption that the unknown error terms can be approximated by a zero mean, normally distributed random variable, so

$$
\mathrm{FLE}_{\mathrm{sys}}=\mathrm{x}_{\mathrm{m}}-\mathrm{x}_{\mathrm{rb}, \text { loo }}+\mathrm{TRE}_{\mathrm{sys}}+\mathrm{TRE}_{\mathrm{ran}}-\mathrm{FLE}_{\mathrm{ran}}
$$

becomes

$$
\mathbf{F L E}_{\mathbf{s y s}}=\mathbf{x}_{\mathbf{m}}-\mathbf{x}_{\mathrm{rb}, \text { loo }}+\mathcal{N}(\mathbf{0}, \sigma)
$$

Whilst equation 14 is of limited use for determining individual values of $\mathbf{F L E}_{\mathbf{s y s}}$ it is ideal for fitting the systematic error function $f_{i}(\theta, \phi, x, y, z, s)$ by minimising the sum of least squares. The positions $\mathbf{x}_{\mathbf{m}}$ and $\mathbf{x}_{\mathrm{rb} \text {,loo }}$ were sampled using data from the composite (22 IRED) rigid body and the systematic error function was fitted to the sampled data.
Equation 14 relies on the assumption that $\mathbf{T R} \mathbf{E}_{\mathbf{s y s}}$ is either small relative to the other error terms or can be modelled as a zero mean random variable. Provided sufficient IREDs are visible this assumption should be valid. Therefore only frames with all 22 active IREDs were used for the curve fitting. The validity of this assumption is tested in the validation process, see section 7.1.

\subsection{Estimation of Independent Random Error}

Given two IREDs $m, n$ on the same rigid body, if $\theta_{m} \approx$ $\theta_{n}, \phi_{m} \approx \phi_{n}, x_{m} \approx x_{n}, y_{m} \approx y_{n}$ and $s_{m} \approx s_{n}$, it follows that

$$
f_{i}\left(\theta_{m}, \phi_{m}, x_{m}, y_{m}, z_{m}, s_{m}\right) \approx f_{i}\left(\theta_{n}, \phi_{n}, x_{n}, y_{n}, z_{n}, s_{n}\right)
$$

If this is true for all IREDs on the rigid body, then all IREDs will be subject to the same systematic error $\mathbf{F L E}_{\text {sys }}$, and $\mathbf{T R E}_{\mathrm{sys}}=\mathbf{F L E}_{\text {sys. }}$. In this situation equation 12 reduces to equation 16 .

$$
\mathbf{F L E}_{\mathrm{ran}}=\mathrm{x}_{\mathrm{m}}-\mathrm{x}_{\mathrm{rb} \text {,loo }}+\mathbf{T R E}_{\mathrm{ran}}
$$

FLE $_{\text {ran }}$ is modelled as an independent random variable, meeting the assumptions required by [17], allowing the use of equation 4 to estimate the covariance of $\mathbf{F L E}_{\mathbf{r a n}}$.

As all the IREDs on the standard (6 IRED) pointer are mounted at the same angle, the requirements for the validity of equation 16 are met. To estimate $\mathbf{F L E}_{\text {ran }}$ the tracking data for the standard pointer was used to generate FRE covariance matrices for each of the 6 IREDs. Equation 4 was then used to estimate covariance matrices for FLE for each of the 6 IREDs. The average of these six matrices was used to model the random error component of equation 5 .

\section{Results}

\subsection{Estimation of Error Functions}

\subsubsection{Estimation of Independent Error}

Covariance matrices for the FLE based on the FRE covariance were calculated as per [17] for the 6 IRED pointer. Figure 6 shows the standard deviations of the FLE in the $x z$ and $y z$ planes. A high degree of anisotropy is present with the standard deviation in the $z$ direction of $0.11 \mathrm{~mm}$ being around 4 times greater than the standard deviation in either the $x$ or $y$ directions.

\subsubsection{Estimation of Bias Function ( $f$ )}

The method described in Section 5.3 was applied to the tracking data for the composite rigid body with 22 IREDs arranged at various angles.

Correlations between the input parameters and the error were searched for using Pearson correlation coefficients and visual examination of error plots. Figure 7 shows a representative selection of plots for error versus angle. Figure 7 show dependencies between the error in the $\mathrm{X}$ direction and the angle to the $\mathrm{YZ}$ plane $(\theta)$, 

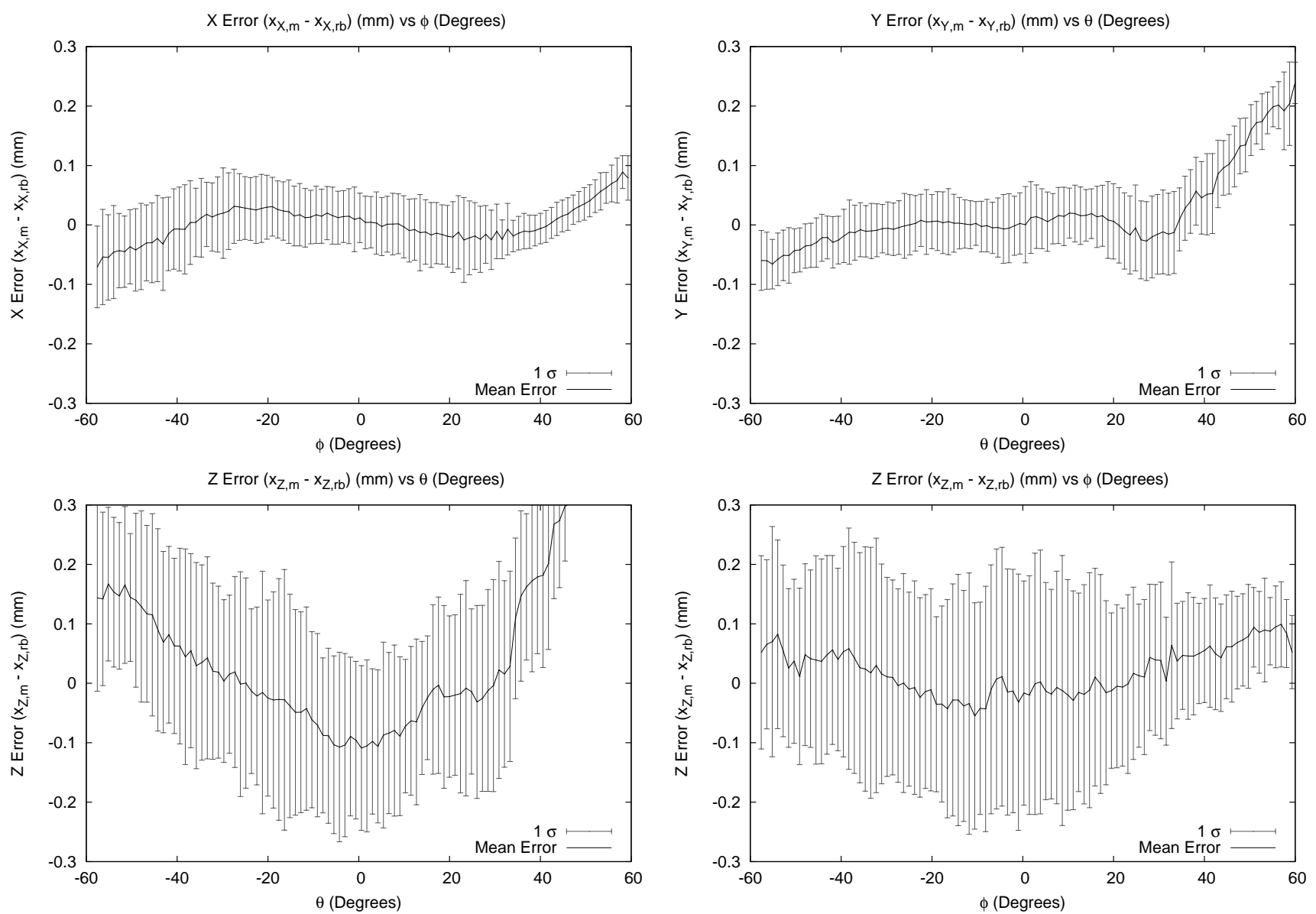

Fig. 7. Plots of directional errors versus IRED pose parameters, as defined on figure 3 . The remaining parameters (IRED positions and speed) did not show any relationship with the errors, therefore they have not been shown here.
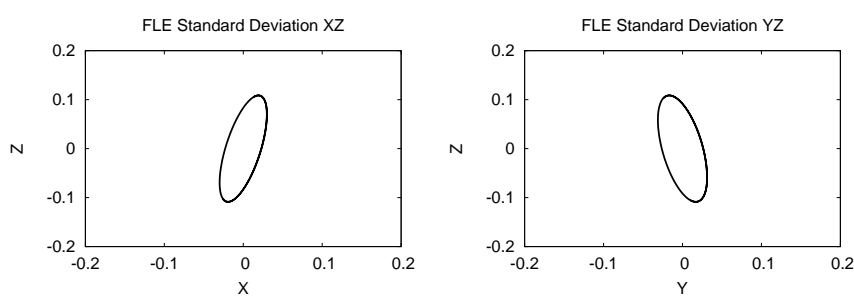

Fig. 6. The standard deviations of the random component of the FLE in the $x z$ and $y z$ planes. The standard deviation in the $z$ direction of $0.11 \mathrm{~mm}$ is significantly greater than the standard deviation in either the $x(0.023 \mathrm{~mm})$ or $y$ (0.026 mm).

the error in the $\mathrm{Y}$ direction and the angle to the $\mathrm{XZ}$ plane $(\phi)$, and the error in $\mathrm{Z}$ direction and both the angles. The remaining parameters from equation $5(x, y, z$ and $s$ ) did not appear to influence the IRED errors, based both on the Pearson correlation coefficient and a visual examination of the error plots. It should be noted that this result does not indicate that these parameters have no effect in the general case, only that any such effect could not be detected using this rigid body. This is discussed further in Section 9.
On the basis of the results shown in Figure 7 the form of Equation 5 was simplified to $F L E_{i}=f_{X, Y, Z}(\theta, \phi)+$ $\mathcal{N}\left(0, \sigma_{X, Y, Z}\right)$ with $f$ modelled as a third order polynomial, see equation 17.

$$
\begin{aligned}
f_{X, Y, Z}= & c_{1, i} \theta^{3}+c_{2, i} \theta^{2}+c_{3, i} \theta+c_{4, i} \phi^{3}+c_{5, i} \phi^{2}+ \\
& c_{6, i} \phi+c_{7, i} \theta^{2} \phi+c_{8, i} \theta \phi+c_{9, i} \theta \phi^{2}+c_{10, i}
\end{aligned}
$$

A third order polynomial model was used as it appears to be the simplest model to fit the data. Use of higher order polynomials would likely result in over fitting to the available data.

Equation 17 was fitted to the error data derived from the composite cross and pointer rigid body. The fitting was performed by minimising the least squares residuals using the Levenberg-Marquardt algorithm implemented in the GNU Scientific Library. Figure 8 shows the resulting fitted functions. Table 3 gives numeric values of the fitted coefficients.

\section{Validation}

\subsection{Validation using Simulated Error Functions}

The accuracy of the error function estimation methods were first validated using simulation of the tracking 

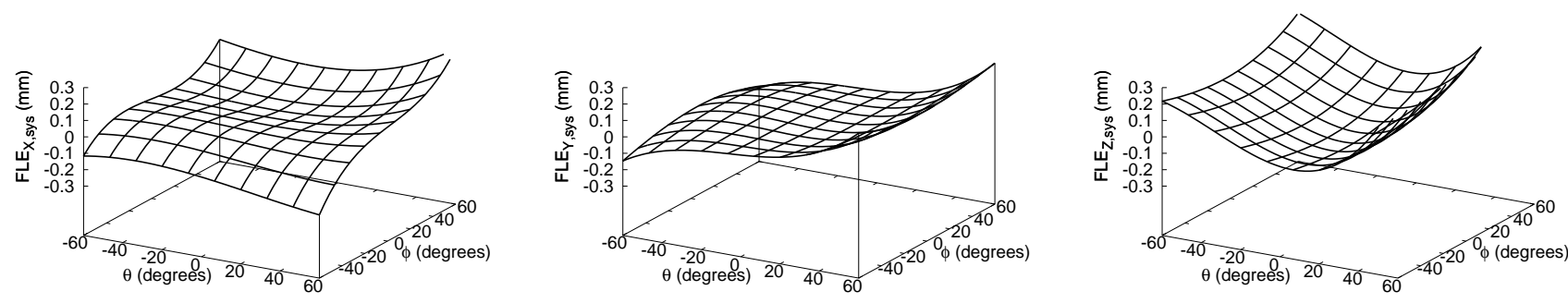

Fig. 8. Polynomial functions (equation 17) fitted to the errors estimated using the composite rigid body. Errors in the $x$ direction are shown at the left, $y$ errors in the middle and $z$ errors at the far right. Similarly to the random component the errors in the $z$ direction are significantly larger than in the $x$ and $y$ directions. The $x$ and $y$ directional errors show $a$ cubic relationship with one of the angles. The $z$ direction errors show a parabolic relationship with both angles.

\begin{tabular}{|l|l|l|l|}
\hline Dir. & $\mathrm{X}$ & $\mathrm{Y}$ & $\mathrm{Z}$ \\
\hline$c_{1, i}$ & $1.8 \times 10^{-7}$ & $7.3 \times 10^{-7}$ & $1.1 \times 10^{-6}$ \\
$c_{2, i}$ & $1.4 \times 10^{-5}$ & $2.0 \times 10^{-5}$ & $1.3 \times 10^{-4}$ \\
$c_{3, i}$ & $8.8 \times 10^{-5}$ & $-4.6 \times 10^{-4}$ & $-2.3 \times 10^{-4}$ \\
$c_{4, i}$ & $-4.6 \times 10^{-4}$ & $-2.9 \times 10^{-3}$ & $-9.5 \times 10^{-2}$ \\
$c_{5, i}$ & $8.8 \times 10^{-7}$ & $-9.4 \times 10^{-8}$ & $1.2 \times 10^{-7}$ \\
$c_{6, i}$ & $-3.0 \times 10^{-6}$ & $-1.8 \times 10^{-6}$ & $2.9 \times 10^{-5}$ \\
$c_{7, i}$ & $-1.4 \times 10^{-3}$ & $-1.4 \times 10^{-4}$ & $5.7 \times 10^{-4}$ \\
$c_{8, i}$ & $4.7 \times 10^{-7}$ & $1.0 \times 10^{-7}$ & $2.8 \times 10^{-7}$ \\
$c_{9, i}$ & $2.1 \times 10^{-5}$ & $-1.4 \times 10^{-6}$ & $2.1 \times 10^{-5}$ \\
$c_{10, i}$ & $-8.3 \times 10^{-8}$ & $3.7 \times 10^{-7}$ & $-5.0 \times 10^{-8}$ \\
\hline
\end{tabular}

TABLE 3

The fitted polynomial coefficients.

process, with the IREDs perturbed by the fitted error function equation 5. Simulated tracking data for both the 6 and 22 IRED rigid bodies was generated by first transforming the respective reference rigid bodies using the rigid body transforms from the actual tracking data. The resulting simulated tracking data mimics the actual tracking data, but with zero FLE. The IREDs are then perturbed using the known error function and the resulting tracking output subjected to the same analysis as for the actual data. The resulting estimates of the systematic and independent errors can be compared to the known input error function.

\subsubsection{Validation of Independent Error Estimate}

The method described in Section 5.5 was used to estimate the random component of the error using a simulated data set based on the 6 IRED pointer. Figure 9 shows the results of this validation procedure. The accuracy of the estimation is similar to the results presented by [17].

\subsubsection{Validation of Bias Function Estimate}

The estimation of the error bias functions was validated using a similar approach to the preceding section. The method described in Section 7.1 was applied to the composite rigid body. Individual IREDs were perturbed according to equation 17 with coefficients taken from tables 3 and 6. The method described in Section 5.5
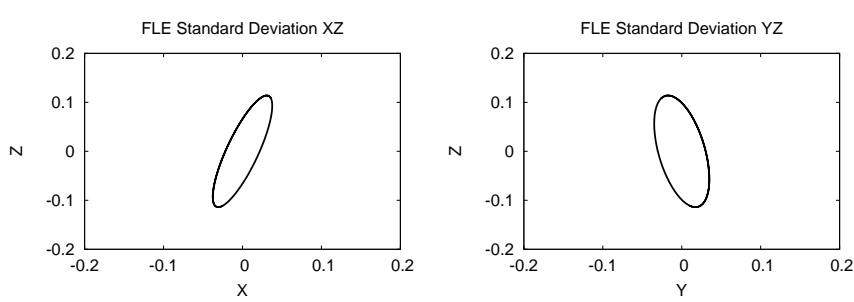

Fig. 9. The standard deviations of the random component of the FLE in the Xz and yz planes. $\sigma_{z}=0.12 \mathrm{~mm}, \sigma_{x}=$ $0.021 \mathrm{~mm}, \sigma_{y}=0.030 \mathrm{~mm}$ The shape and magnitude of the estimated standard deviations are very close to the input functions, Figure 6.

\begin{tabular}{|l|l|l|l|}
\hline Error & $\mathrm{X}(\mathrm{mm})$ & $\mathrm{Y}(\mathrm{mm})$ & $\mathrm{Z}(\mathrm{mm})$ \\
$\left\langle\left|F L E_{\text {Act. }}\right|\right\rangle$ & 0.0407 & 0.0436 & 0.1370 \\
\hline$\left\langle\left|F L E_{\text {Est. }}\right|\right\rangle$ & 0.0402 & 0.0350 & 0.0991 \\
\hline$\left\langle\left|F L E_{\text {Est. }}-F L E_{\text {Act. }}\right|\right.$ & 0.0076 & 0.0101 & 0.0446 \\
\hline Percentage Error & $-18.7 \%$ & $-23.2 \%$ & $-32.6 \%$ \\
\hline
\end{tabular}

TABLE 4

The result of integrating the difference between the error functions shown in Figure 10 between $\pm 60^{\circ}$ in both directions. Despite capturing the general shape of the error functions the method still underestimates the systematic errors by around $25 \%$.

was applied to the simulated data to estimate the error functions. The error is represented pictorially in Figure 10 and numerically in table 4 . The method does capture the general shape of the systematic error functions, though does underestimate the systematic errors. To put a numerical value on the amount of underestimation the two surfaces were integrated over the range of angles $\pm 60^{\circ}$ and the enclosed volume expressed as a percentage of the actual error.

\subsection{Validation using Pointer Calibration Data}

As the number of visible IREDs on a pointer is reduced, the tip localisation error will increase. A corresponding increase in the calibration error $E_{C a l}$ would be expected. 

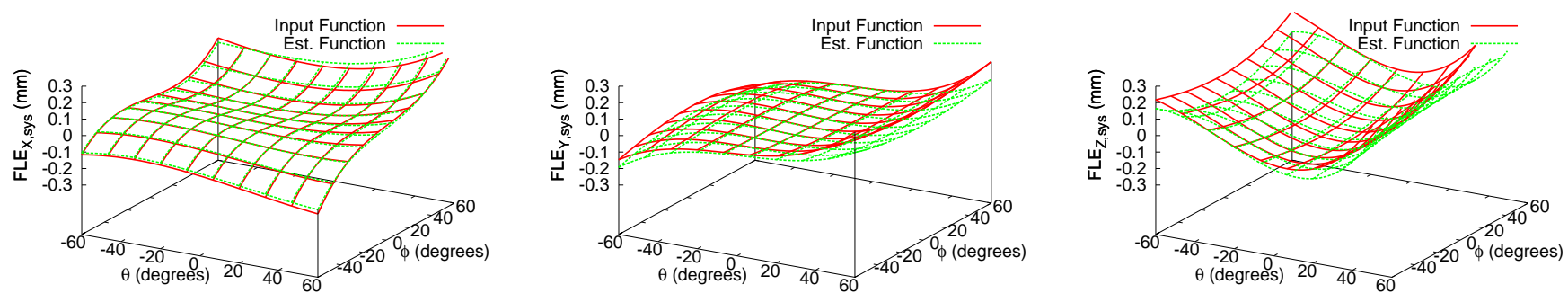

Fig. 10. The known input error function and the error function estimated using simulated measurements. The proposed method is largely successful in estimating the shape of the error functions.

This effect can be observed on the 22 IRED pointer by progressively masking IREDs and repeating the calibration. For real calibration data it is not practical to know the actual pointer tip position $\mathbf{p}_{\mathrm{gs}}$, however, if the calibrated pointer tip when all IREDs are visible is used as a pseudo gold standard position, it is possible to show the change in calibrated tip position as the IRED visibility is changed. The same procedure can be performed using simulated data and various models of FLE. A good model of the FLE should provide a good description of the observed ressults.

A subset of the calibration tracking data for the composite rigid body, see section 4, consisting only of frames (1107 frames) with all 22 IREDs visible was used. Calibration data for pointers with 3 to 21 IREDs visible were generated by masking the data using patterns of IRED visibility that had been observed in the original data set. The resulting 20 data sets were used to perform a pointer calibration, giving 20 estimates of the pointer tip position. The pointer tip position found using all 22 IREDs is then used as a gold standard to calculate $E_{C a l}$ for the remaining IRED visibilities.

To simulate the calibration process a set of 1107 synthetic data frames with zero FRE was created by transforming the reference rigid body into the Optotrak coordinate system using the pointer poses observed for the real data. Each of these 1107 frames was then translated to place the pointer tip at $q$, i.e. generating a perfect calibration with zero calibration error $\left(E_{C a l}\right)$. The individual IREDs were then perturbed using the derived error model, equation 17, to generate a simulated data set. The calibration and IRED masking process described above was used to generate a corresponding set of simulated $E_{C a l}$ s for reducing IRED visibilities.

The calibration simulation was repeated with three models of independent FLE, based on the literature. Homogeneous and heterogeneous models of independent anisotropic error, and homogeneous isotropic error. For the anisotropic errors covariance matrices for the FLE were computed as per [17], using all 1107 frames of data. Equation 3 was used to estimate the expected absolute value of the isotropic error.

Figure 11 shows the change in the estimated $E_{C a l}$

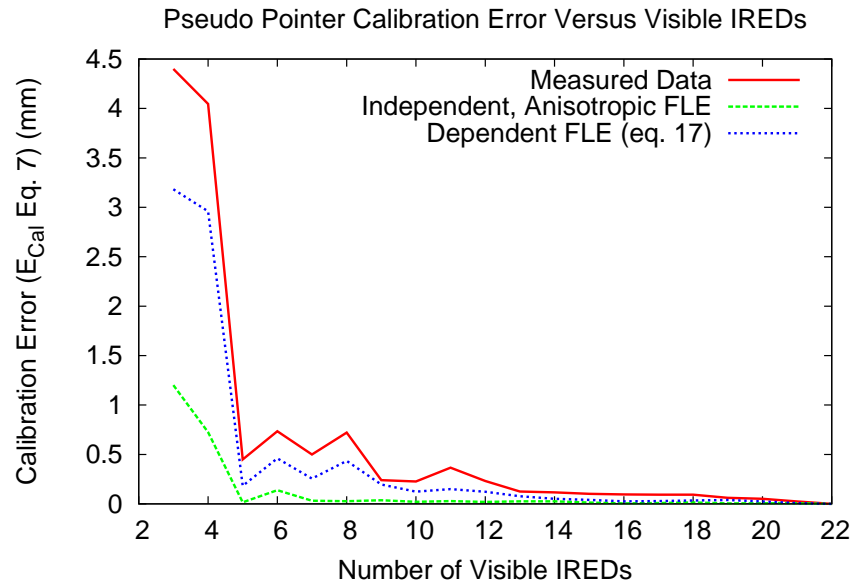

Fig. 11. Measured and modelled values of pointer calibration error, estimated using pointer tip position calculated using all 22 IREDs as pseudo gold standard. The proposed two component FLE model gives a result substantially closer to the observed results than any of the models using only independent FLE. Only the results for homogeneous anisotropic noise are shown here for clarity, as neither homogeneous isotropic nor heterogeneous anisotropic FLE gave an improved result.

for reducing IRED visibility, for both the measured and simulated data. The proposed error model, combining systematic and independent errors, is shown to give a much closer approximation of the observed results than any of the independent error models. Only the results for homogeneous anisotropic noise are shown as this was the best performing of the independent error models.

Having an estimate of the systematic component of the FLE, the logical extension is to try and correct for the systematic error in the registration process. This can be done at a small computational cost, by using an initial estimate of the IRED surface normal angles to calculate the systematic error, correcting the measured IRED position, and repeating the registration. Figure 12 shows the measured change in estimated $E_{C a l}$ with and without correction for systematic error. There is a marked improvement in the calibration results for low 


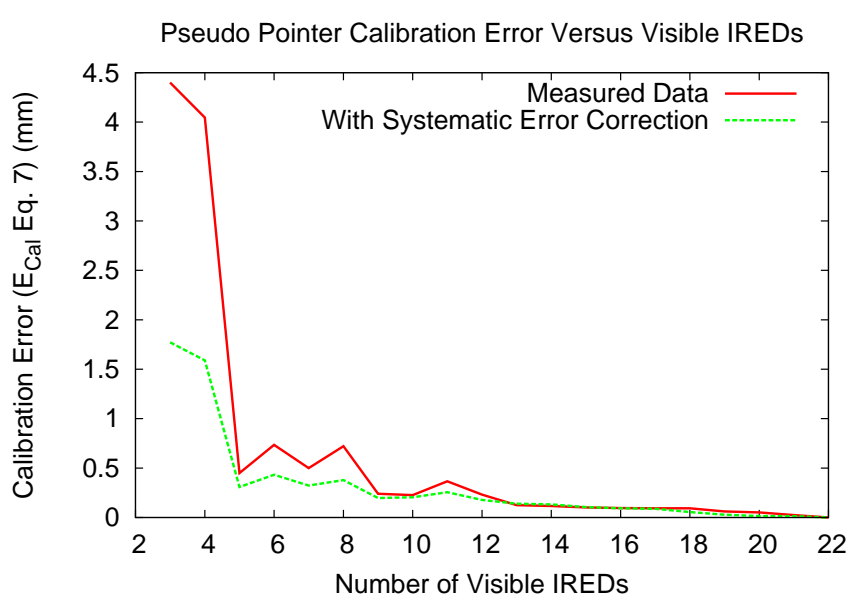

Fig. 12. Measured values of pointer calibration error with and without correction for systematic error in the registration process. Correcting for the systematic error shows a marked reduction in the estimated calibration error at low IRED visibility.

IRED visibility.

\section{Applications}

\subsection{Calibration Simulation}

In practice it is not possible to know the actual calibration error, $E_{C a l}$, for a given pointer calibration procedure. A realistic model of FLE enables simulation of the pointer calibration process to give estimates of the expected pointer tip spread and the calibration error. In this section the calibration process is simulated using three models of FLE. one application for modelling of the FLE is the simulation of the calibration process in to estimate the expected value of calibration error. In this section the pointer calibration process is simulated using three different models of FLE.

The rigid body geometry $X_{r}$, the location of the pointer tip in pointer coordinates ( $\mathbf{p}$ ) and in world coordinates (q) were taken from the actual pointer calibration for the 6 IRED pointer. Each calibration simulation was performed using 100 pointer poses selected at random from the 5838 poses recorded for the 6 IRED pointer, see section 4. As in Section 7.2 "perfect" calibration sets were created by translating the pointer to give zero pointer tip spread, then the IRED positions were perturbed using three models of FLE. The simulated tracking data are used to perform a pointer calibration as per section 5.2. For each model of FLE the calibration simulation was repeated 1000 times to give a reasonable population of results.

The first model of FLE is the two component model proposed in this paper, i.e. equation 17. The remaining 2 methods use the more common approach of modelling the FLE as an independent and anisotropic normal distribution. The FLE covariance matrices for each of the models are determined using the method of [17]

\begin{tabular}{|l|l|l|l|}
\hline FLE Model & $\left\langle F L E_{X} \mid\right\rangle$ & $\left\langle\left|F L E_{Y}\right|\right\rangle$ & $\left\langle\left|F L E_{Z}\right|\right\rangle$ \\
\hline 1: Equation 17 & 0.038 & 0.036 & 0.111 \\
2: [17] 22 IRED Pointer & 0.029 & 0.024 & 0.172 \\
3: [17] 6 IRED Pointer & 0.024 & 0.023 & 0.081 \\
\hline
\end{tabular}

TABLE 5

The calibration simulation uses three models of FLE. Model 1 uses the error model put forward by this paper (equation 17). The remaining two models use an independent random error, estimated using the FRE covariance matrices from a set of tracking data, [17]. Model 2 uses the FRE from the 22 IRED composite pointer, model 3 used the FRE from the 6 IRED pointer. The table shows the expected absolute values of FLE for each model.

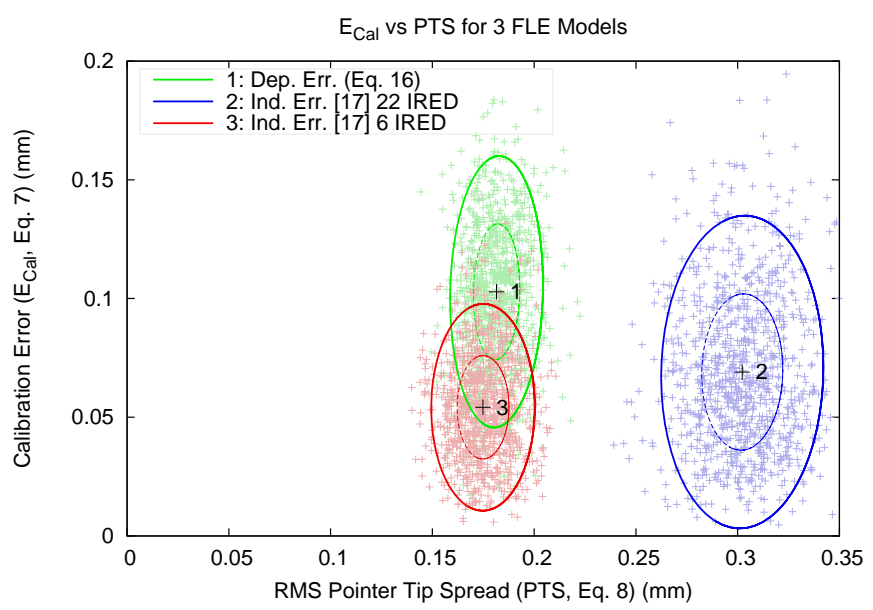

Fig. 13. Plots of the RMS pointer tip spread ( $P T S$, equation 9) and the actual calibration error ( $E_{C a l}$, equation 8) for each of the three models of FLE, defined in Table 5. The RMS pointer tip spread is linked to the expected absolute value of the random component of the FLE. Hence models 1 and 3 , which have the same random component of FLE, have similar pointer spread values. The actual calibration error however is significantly changed when the systematic error is introduced (model 1 ). The calibration error for model $1(0.1 \mathrm{~mm})$ is double that of model 3 $(0.05 \mathrm{~mm})$.

(equation 4) to estimate the FLE covariance from the FRE covariance. Model 2 uses the composite (22 IRED) rigid body to estimate the FRE covariance matrices. Model 3 uses the standard (6 IRED) pointer to estimate the FRE covariance matrices. As discussed in section 5.5 using the method from [17] on the flat (6 IRED) pointer fails to capture the angle dependent error, resulting in significantly smaller estimated FLE. The three models and their expected absolute errors are listed in Table 5. Figure 13 plots the RMS pointer tip spread against the actual calibration errors for each simulated model.

Three important results are evident from Figure 13. Firstly, within each data set there is no correlation between the pointer tip spread and the actual calibration 
error, i.e. the calibration accuracy for a given calibration cannot be predicted from the pointer tip spread. This result is widely known but often overlooked in practice. Secondly the expected calibration error when using the formula (model 1) is approximately double that using any of the other models. That is, the assumption of independence between the individual FLEs significantly underestimates the actual calibration error. The last result of note is that for the cases with independent error, whilst the magnitude of the error has a strong effect on the pointer tip spread, the effect on the expected calibration error is minimal. The last two points are due to the fact that the calibration method itself is based on the assumption that the marker errors are independent.

\subsection{Extension to Laparoscope Tracking}

The preceding results have an important application to laparoscope tracking. Due to the physical restrictions present in laparoscopic surgery it is not possible to place the IREDs near the target to be tracked (the tip of the laparoscope). For this reason the tracking errors are in general quite high as any error in estimating the angular orientation of the laparoscope is amplified by the length of the laparoscope body.

To demonstrate the effects of this the following simulation was performed. Two realistic laparoscope tracking configurations were simulated, both with 12 IREDs mounted on a collar at the distal end of the laparoscope. In one case the 12 IREDs are all mounted with the same orientation, so angle dependent errors will be coherent, as was the case for the 6 IRED pointer. In the other case the orientation of the IREDs is varied, as might be done in the hope of getting a greater tracking range. For simplicity the two collars will henceforth be referred to as the "flat" and "bumpy" collars respectively. Figure 14 shows the two configurations. Laparoscope tracking is then simulated for each configuration under two models of tracking error, one using the proposed error formula (equation 17), and the other using independent random derived using the method of [17] from the composite rigid body, (Model 2, from Table 5). Figure 15 defines the laparoscope geometry.

The laparoscope tracking simulation is performed as follows. The laparoscope and the target point are first transformed to the Optotrak's coordinate system into a physically realistic pose. A range of laparoscope poses are used simulating the laparoscope pivoting about a trocar point across an angle of approximately 120 degrees in both the $X$ and $Y$ directions. The IREDs are then perturbed using either the error formula or an independent random variable.

An important aspect of laparoscope tracking is the fact that in practice IREDs will be obscured. This may be due to the position of the laparoscope itself, for example if the laparoscope is positioned at an angle to the tracking cameras the laparoscope itself will obscure the IREDs on one side of the tracking collar. In other cases some
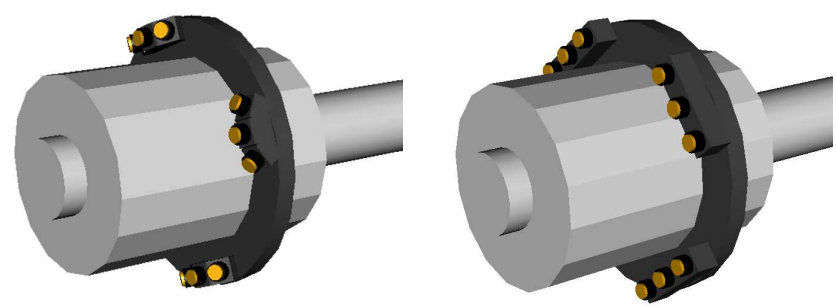

Fig. 14. The two IRED configurations tested. Both configurations have the IREDs in identical positions. 12 IREDs are mounted in groups of three around the perimeter of the laparoscope camera unit. This configuration, while not ideal, has been used in practice and found to be practical. The collar on the left, henceforth known as the "bumpy" collar, has the two outer IREDs in each group of 3 angled outwards at 30 degrees. Such a configuration might be used to maximise the chance of at least some IREDs being visible at extreme angles. The collar on the right, the "flat" collar, is identical, but with all 12 IREDs having identical surface normals.

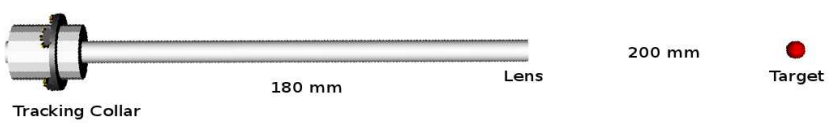

Fig. 15. The geometry of the laparoscope tracking model. The laparoscope modelled has a distance of $180 \mathrm{~mm}$ from the tracking collar to the lens. The surgical target is a further $200 \mathrm{~mm}$ in front of the laparoscope lens. Though the pose of the laparoscope changes during the simulation to achieve different IRED normal angles, the position of the target remains fixed with respect to the laparoscope lens, i.e. the target moves relative to the coordinates of the tracking camera system or "operating room".

IREDs may be obscured by one of the many people in the operating theatre or other equipment. One way to address this is to only use frames when all IREDs are visible, this however somewhat compromises the tracking systems utility. It would be far better to design the tracking collar to be robust to obscured IREDs. To account for this in the simulation a subset of IREDs are then turned off. The IREDs were turned off either individually or in physically realistic groups, i.e. on one side of the tracking collar or physically adjacent IREDs.

The positions of the visible and perturbed IREDs were then used to estimate the position and pose of the laparoscope. The target point was then projected onto a plane $200 \mathrm{~mm}$ distant to and parallel to both the known position of the laparoscope and the estimated position of the laparoscope. The distance between the two points was recorded and the simulation was repeated with a new laparoscope pose. The simulation was repeated approximately 6000 times and the RMS projected error 


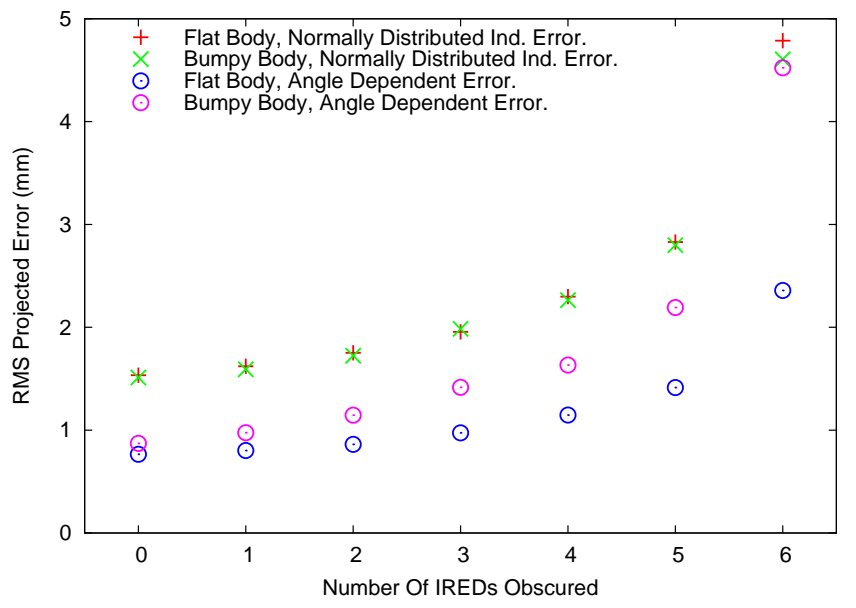

Fig. 16. The on screen tracking error for a point 200 $\mathrm{mm}$ distant from the endoscope lens. Two different models of FLE are used, independent normally distributed anisotropic noise (model 2, table 5), and an angle dependent systematic error added to a normally distributed noise term (model 1, table 5). A rigid body with uniform IRED surface normals (flat) and another with varying (bumpy) IRED surface normals were simulated. For independent FLE IRED orientation has no effect on projected error. When a systematic error is introduced the effect of IRED orientation becomes important. With 6 IREDs obscured the flat rigid body gives a projection error $2 \mathrm{~mm}$ less than the bumpy rigid body.

recorded. The process was repeated for each model of FLE, for each IRED geometry, and different numbers of obscured IREDs, between 0 and 6 . The results are shown in Figure 16.

The independent error model gives a significantly higher back projection error than the systematic error model. The results for the systematic error model shows that IRED orientation can have a significant effect on the tracking performance. When all IREDs are visible the IRED orientation does not have a significant effect. However as IREDs are obscured, as will occur in practice, the results diverge. In this case having all the IREDs at the same angle results in a system that is significantly more robust to loss of IRED visibility. When 6 IREDs are obscured the flat rigid body gives a projection error around $2 \mathrm{~mm}$ smaller than the bumpy rigid body.

Because the errors are coherent, the systematic tracking error will result in a translational error at the laparoscope collar with an expected absolute value less than $0.1 \mathrm{~mm}$. Unlike rotational errors, translational errors at the collar will not be magnified by the lever arm of the laparoscope. A $0.1 \mathrm{~mm}$ translational error at the collar results in a $0.1 \mathrm{~mm}$ translational error at the laparoscope lens, which is insignificant in comparison to the errors at the laparoscope lens due to rotational errors.

To test for the existence of a limiting laparoscope length, where a bumpy collar may outperform a flat collar, the simulation was repeated using targets at shorter

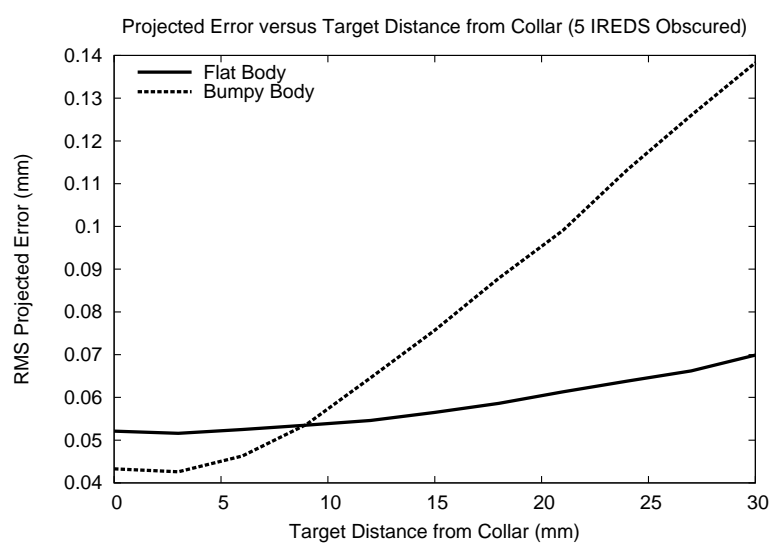

Fig. 17. The projected error for a laparoscope as a function of the distance of the target from the centroid of the tracking collar. A flat collar gives a higher error at points very close $(<10 \mathrm{~mm})$ from the collar. However as the distance from the collar increases the flat collar significantly out performs the bumpy collar. The crossing point of the two curves at less than $10 \mathrm{~mm}$ from the collar centroid indicates that for all practical tracking problems a rigid body with the IREDs arranged at the same angle is preferable.

distances (between 0 and $30 \mathrm{~mm}$ ) from the tracking collar centroid. To simplify the illustration only the results for 5 visible IREDs were plotted. Figure 17 shows the resulting projection errors.

The errors at distance $0 \mathrm{~mm}$ in Figure 17 represent the translational component of the registration errors. It is apparent that the bumpy collar has a smaller translation error than the flat. However, as the distance from the centroid increases it is the rotational component of the error that becomes significant. In fact the rotational error starts to dominate at distances greater than $10 \mathrm{~mm}$, which is an order of magnitude less than the distances encountered in practice. In all practical cases then, a flat collar would be expected to outperform a bumpy collar.

\section{Discussion}

This paper starts with the assumption that the tracking error for each IRED can be divided into a repeatable systematic error and a non repeatable random error. Methods for determining each error are then presented. The method for estimating the random component is taken from [17].

The method for determining the systematic error is straightforward yet novel. The method has the advantage that it does not rely on any knowledge of the physics of the underlying system. It is not necessary to know the physical characteristics of the tracking system, i.e. number of camera(s), focal lengths, positions of cameras, etc. The same method could in theory be applied easily to other point based tracking systems. However this freedom has probably come at the cost of accuracy. It seems likely that if the data had been fitted to some 
underlying physical model of the system, such as done by [20], the results may have been improved.

The resultant 2 component model of FLE marks a significant improvement on the classical model of independent, normally distributed FLE. The proposed model was used to model the change in the pointer tip calibration error with reduced IRED visibility (figure 11) and shown to provide a much closer match to observed results than existing independent error models.

The two component model was then used in two simulations to demonstrate cases where the use of existing independent error models can lead to significant errors. Simulations of the pointer calibration (figure 13) showed that independent error models can underestimate the pointer calibration error by a factor of 2 . Simulations of laparoscope tracking using arrangements of IREDs with homogeneous and inhomogeneous IRED surface normals also gave an interesting and significant result, see figure 16. When a significant systematic error is present, arranging the tracking IREDs such that the systematic error is the same for all IREDs gives a significantly lower tracking error at the pointer (or camera) tip. This is due to the fact that such an arrangement results in a purely translational error for the systematic component of FLE. Unlike rotational errors, translational errors are not amplified by the long lever arm of a laparoscopic tool or camera. This effect was true for tool lengths greater than $10 \mathrm{~mm}$.

\subsection{Future Work}

No correlations were found between IRED position or speed. Such correlations may exist, however the rigid bodies used in this paper would be unable to detect them. In order to detect any positional correlations using the method presented a significantly larger (with an area of approximately $1 \mathrm{~m}^{2}$ ) rigid body would be required. This would be difficult to achieve in practice, but may be useful for some applications. Similarly, by sampling the rigid bodies used in this paper at a larger range of speeds it may be possible to find a change in the random error component, as observed by [15].

The almost universally used registration method presented in Section 5.1 is based on the assumption of isotropic, homogeneous, independent, and normally distributed marker error, an assumption shown to be wrong in this case. Methods have been proposed in the literature to solve the weighted weighted Procrustes problem [21], [14], [6], [22]. These can account for the anisotropy of the error, but not the fact that the errors are not independent. The availability of a more realistic model for FLE will enable the better assessment of these and other registration methods.

As detailed in section 7.1.2 the method used to estimate the systematic error function underestimates the size of the systematic error by around $30 \%$. One source of error is the assumption made in Section 5.4 that the systematic TRE $\left(\mathbf{T R E}_{\text {sys }}\right)$ can be modelled as a zero mean random variable. It is unlikely that this assumption is valid and further work would help to determine the effect of this assumption. One possibility for correcting this error would be to use an iterative approach to solve equation 12.

\section{Conclusion}

To bring image guided surgery to laparoscopic procedures will require accurate tracking of laparoscopic tools and cameras. In order to accurately track laparoscopic cameras and tools an improved understanding of the tracking system error is required. This paper has presented a simple method, that could be implemented by most tracking system users, to estimate the error functions of a given tracking system. The method is particularly applicable to systems using active IRED markers but could also be applied to systems using passive markers, though the results would be expected to be quite different.

The system is not entirely accurate, but in its current form can be used to improve the design of laparoscopic tracking collars. Most significantly it has been shown that there are very significant benefits to using a pattern of IREDs with homogeneous surface normal directions.

Image guided surgery using point based tracking systems is becoming increasingly commonplace. The widely used assumption that the target registration error can be modelled as an independent normally distributed random variable can lead to some errors being underestimated by a factor of 2 . Furthermore, a proper understanding of the tracking error enables significantly improved tracking system design.

\section{ACKNOWLEDGMENTS}

\section{REFERENCES}

[1] L. Adhami and E. Coste-Maniére, "A versatile system for computer integrated mini-invasive robotic surgery," in Medial Image Computing and Computer Assisted Intervention, ser. LNCS, T. Dohi and R. Kikinis, Eds., vol. 2488. Springer-Verlag, 2002, pp. 272281.

[2] J. M. Fitzpatrick, J. B. West, and C. R. Maurer Jr, "Predicting error in rigid-body, point-based registration," IEEE Transactions on Medical Imaging, vol. 17, no. 5, pp. 694-702, October 1998.

[3] A. D. Wiles, D. G. Thompson, and D. D. Frantz, "Accuracy assement and interpretation for optical tracking systems," in SPIE Medical Imaging, Visualisation, Image-Guided Procedures, Display, vol. 5367, 2004, pp. 421-432.

[4] NDI, Optotrak Certus Specifications, NDI, Ontario, 103 Randall Drive. Waterloo, Ontario, Canada, N2V 1C5, 1992.

[5] J. B. West and C. R. Maurer Jr, "Designing optically tracked instruments for image-guided surgery," IEEE Transaction on Medical Imaging, vol. 23, no. 5, pp. 533-545, 2004.

[6] P. G. Batchelor and J. M. Fitzpatrick, "A study of the anisotropically weighted procrustes problem," in MMBIA '00: Proceedings of the IEEE Workshop on Mathematical Methods in Biomedical Image Analysis. Washington, DC, USA: IEEE Computer Society, 2000, pp. 212-218.

[7] W. Birkfellner, M. Figl, K. Huber, F. Watzinger, F. Wanschitz, J. Hummel, R. Hanel, W. Greimel, P. Homolka, R. Ewers, and H. Bergmann, "A head-mounted operating binocular for augmented reality visualization in medicine - design and initial evaluation," IEEE Transactions on Medical Imaging, vol. 21, no. 8, pp. 991-997, 2002. 
[8] A. P. King, P. J. Edwards, M. R. Pike, D. L. G. Hill, and D. J. Hawkes, "An analysis of calibration and registration errors in an augmented reality system for microscope-assisted guided interventions," in Medical Image Understanding and Analysis, 1999. [Online]. Available: citeseer.ist.psu.edu/213204.html

[9] P. J. Edwards, A. P. King, C. R. Maurer, D. A. de Cunha, D. J. Hawkes, D. L. G. Hill, R. P. Gaston, M. R. Fenlon, A. Jusczyzck, A. J. Strong, C. L. Chandler, and M. J. Gleeson, "Design and evaluation of a system for microscope-assisted guided interventions (magi)," IEEE Transactions on Medical Imaging, vol. 19, no. 11, pp. 1082-1093, 2000

[10] A. D. Wiles, A. Likholyot, D. D. Frantz, and T. M. Peters, "A statistical model for point-based target registration error with anisotropic fiducial localizer error," IEEE Transactions on Medical Imaging, vol. 27, no. 3, pp. 378-390, 2008.

[11] M. H. Mohghari and P. Abolmaesumi, "Distribution of target registration error for anisotropic and inhomogeneous fiducial localization error." IEEE Transactions on Medical Imaging, vol. 28, no. 6, pp. 799-813, June 2009.

[12] A. Danilchenko and J. M. Fitzpatrick, "General approach to firstorder error prediction in rigid point registration," IEEE Transactions on Medical Imaging, vol. 30, pp. 679-693, 2011.

[13] R. Shahidi, M. R. Bax, C. R. Maurer, J. A. Johnson, E. P. Wilkinson, B. Wang, J. B. West, M. J. Citardi, K. H. Manwaring, and R. Khadem, "Implementation, calibration and accuracy testing of an image-enhanced endoscopy system." IEEE Transactions on Medical Imaging, vol. 21, no. 12, pp. 1524-1535, Dec 2002.

[14] A. Danilchenko, A. D. Wiles, R. Balachandran, and J. M. Fitzpatrick, "Improved method for point-based tracking," in Medical Image Computing and Computer-Assisted Intervention, ser. LNCS, T. Jiang, N. Navab, J. P. Pluim, and M. A. Viergever, Eds., vol. 6363. Springer, 2010, pp. 587-594.

[15] P. Barnes, C. Baldock, S. Meikle, and R. Fulton, "Benchmarking of a motion sensing system for medical imaging and radiotherapy," in IEEE Nuclear Science Symposium Conference Record, vol. 6, 2007, pp. 4513-4520.

[16] M. Bauer, M. Schlegel, D. Pustka, N. Navab, and G. Klinker, "Predicting and estimating the accuracy of n-occular optical tracking systems," in Proc. IEEE/ACM Int. Symp. Mixed and Augmented Reality ISMAR 2006, 2006, pp. 43-51.

[17] A. D. Wiles and T. M. Peters, "Real-time estimation of FLE statistics for 3-D tracking with point-based registration," IEEE Transactions on Medical Imaging, vol. 28, no. 9, pp. 1384-1398, 2009.

[18] P. Schönemann, "A generalized solution of the orthogonal procrustes problem," Psychometrika, vol. 31, pp. 1-10, 1966, 10.1007/BF02289451. [Online]. Available: http://dx.doi.org/10.1007/BF02289451

[19] J. M. Fitzpatrick, D. L. G. Hill, and C. R. Maurer Jr, Handbook of Medical Imaging. SPIE Press, 2000, vol. II, ch. Image Registration.

[20] T. Sielhorst, M. Baur, O. Wenisch, G. Klinker, and N. Navab, "Online estimation of the target registration error for n-ocular optical tracking systems," in Medical Image Computing and Computer Aided Interventions, ser. LNCS, vol. 4792. Springer, 2007, pp. 652-659.

[21] R. Balachandran and J. M. Fitzpatrick, "Iterative solution for rigid-body point-based registration with anisotropic weighting (proceedings paper)," in Medical Imaging 2009: Visualization, ImageGuided Procedures, and Modeling SPIE, M. I. M. K. H. Wong, Ed., 2009.

[22] M. T. Chu and N. T. Trendafilo, "On a differential equation approach to the weighted orthogonal procrustes problem," Statistics and Computing, vol. 8, pp. 125-133, 1998. 